\title{
CASA APESTEGUIA: LA CAPACIDAD DE RECICLAJE DE UN EDIFICIO EN EL TIEMPO
}

\section{APESTEGUIA HOUSE: THE CAPACITY OF RECYCLING OF A BUILDING OVER TIME}

\section{Alexander Gálvez Nieto}

\section{Resumen}

El presente artículo trata sobre la relación de la estructura y la espacialidad de un edificio como base para determinar la capacidad de reciclaje del mismo. El caso estudio seleccionado fue la casa Apesteguía, ubicada en Barranco (Lima), cuya estructura permitió diferentes usos, que se cumplieron desde finales del siglo XIX hasta la actualidad, y que promete seguir durando en el tiempo convirtiéndose en un ejemplo de proyecto de reciclaje. El proyecto demuestra que el reciclaje arquitectónico no es una estrategia de moda, producto de la sostenibilidad, sino que es una necesidad tan antigua como la arquitectura misma.

Palabras clave: capacidad de reciclaje; adaptabilidad; durabilidad; espacialidad; estructura.

\section{Abstract}

This article deals with the relation of the structure and the spatiality of a building as a base to determine he recycling capacity thereof. The study case selected was the Apesteguia House, located in Barranco (Lima), whose structure allowed different uses, which were fulfilled from the end of the nineteenth century to the present, and which promises to continue to last in time becoming an example of a recycling project. The project shows that architectural recycling is not a fashionable strategy, a product of sustainability, but a need as old as architecture itself.

Key words: Recycling capacity; adaptability; durability; spatiality; structure. 


\section{Introducción}

La casa Apesteguía fue originalmente un rancho de un piso de adobe que, tras muchas transformaciones, se ha convertido en las oficinas de la agencia de publicidad Fahrenheit DDB. La durabilidad en el tiempo constituye uno de los principios indispensables de un edifico sostenible. Su estudio es de relevancia, puesto que la calidad arquitectónica de la casa exigió su permanencia en el lugar, revelando su capacidad de reciclaje en el tiempo.

\section{Elemento contenedor y espacio conte- nido}

El reciclaje arquitectónico es considerado en la actualidad como una estrategia de sostenibilidad, pues aminora el impacto de la arquitectura en el entorno al buscar la reutilización de las infraestructuras existentes (Martínez, 2012); sin embargo, su práctica es tan antigua como la propia arquitectura (Chacón, 2008; Valdivia, 2008). La capacidad de adaptación a otros usos para futuras generaciones permite al edificio durar en el tiempo, explotando la calidad estructural y espacial del mismo. (Callejo, 2014; Jourda, 2009; Valdivia, 2008). El reciclaje arquitectónico "implica idear nuevas respuestas" para edificios obsoletos o en desuso (Martucelli, 2008, p. 94).

Rondinel (2008) identificaba dos elementos en un edificio en desuso:

- $\quad$ El elemento contenedor (lo físico): compuesto por la estructura, la envolvente, los servicios y los tabiques (Callejo, 2014). Son materiales tangibles sobre los que se pueden aplicar procesos de reutilización o reciclaje.

- El espacio contenido: El reciclaje del espacio está vinculado con el uso que se le puede estar dando y el uso propuesto.

El espacio en sí mismo no cumple ciclos de vida, quien lo hace es la forma de cómo se usa el espacio, por eso primero debe ejecutarse la evaluación del espacio contenedor que contiene el espacio a ser reciclado.

Para Callejo (2014), la relación entre dichas partes mostraría la capacidad de reciclaje del edificio: deben ser convertibles, desmontables, disgregables, expandibles y flexibles (Rondinel, 2008). Si se consiguiese una independización total de las partes del edificio, sería lo más óptimo, dado que cada uno de los elementos podría ser modificado sin afectar a los otros, suponiendo el debido ahorro energético y económico que implica.

Cada una de estas partes deben ser analizadas por separado, desde las capas más internas hacia las más externas, ya que si la estructura no está en buen estado, y no se puede recuperar, se debe pasar a la demolición del mismo. Si en el espacio contenedor el estado de la estructura es aceptable, además de un adecuado espacio contenido, entonces se puede reciclar el proyecto.

La capacidad de reciclaje de un edificio está ligada a la predisposición inicial de sus partes, la modulación en la 
configuración garantiza que se pueda reutilizar en la misma obra. La elaboración de una buena estructura independizada, pensada para crear flexibilidad espacial sería el primer paso para la capacidad de reciclaje.

\section{La estructura y espacialidad de la casa Apesteguia}

La casa Apesteguía, ubicada en el distrito de Barranco, departamento de Lima, Perú, fue originalmente un ran- cho de un piso, construido de adobe, que luego se transformaría en un conjunto bifamiliar de renta (1904). Tal fue su condición, hasta que en 1986 el arquitecto Juvenal Baracco interviene la casona transformándola en 8 departamentos dúplex con mezanines y una tienda museo en el sótano. Posteriormente, el conjunto se convirtió en un hotel que estuvo semiabandonado, hasta que lo compró la agencia de publicidad Fahrenheit DDB, en 2015 (Figura 01).

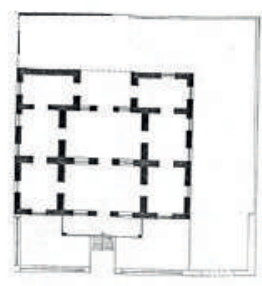

Planta 1(Eatimacióndelautor)

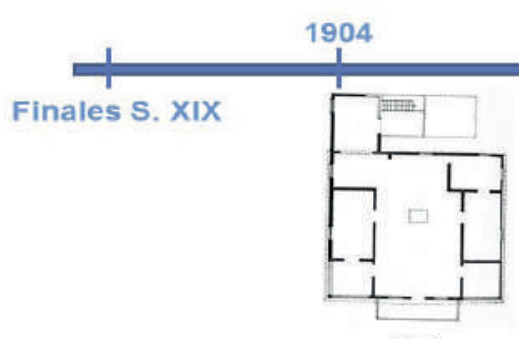

Planta2

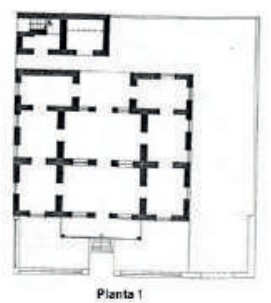

1904

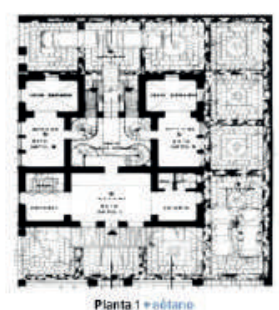

Planta 1 vadtane
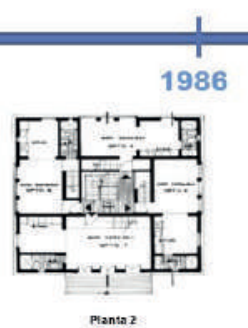

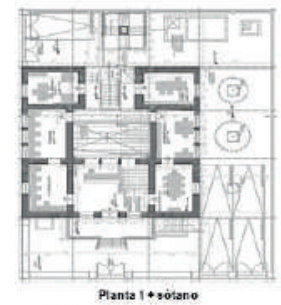

2015

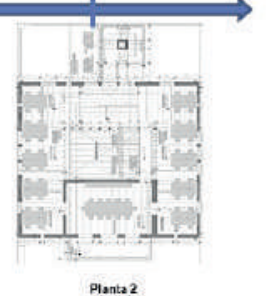

\section{Evolución de la estructura y el espacio de la Casa Apesteguia}

Figura 01. Intervenciones fisicas en la Casa Apesteguía desde finales del S. XIX hasta 2015

Este proyecto de oficinas fue encargado a los arquitectos Maya Ballen y Peter Seinfeld, y fue ganador internacional de la XX Bienal Panamericana de Arquitectura de Quito en la categoría Rehabilitación y Reciclaje. El diseño partió de la premisa de que la pro- puesta se convertiría en una capa más de historia, "producto de múltiples remodelaciones a lo largo de los años" en el edificio (Masunoestudio, 2017).

A través del tiempo, la estructura de adobe del espacio contenedor presentó una modulación que se mantu- 
vo, mientras que el espacio contenido se pudo adaptar mejor a los usos que contuvo debido a la flexibilidad, desmontabilidad y expandibilidad de los materiales de los elementos constructivos, como la quincha, la madera y el fierro. En los muros de adobe de la primera planta se cerraron algunos vanos y se abrieron otros debido a los cambios de uso que se suscitaron, pero ninguna de estas acciones comprometió la calidad estructural de los muros (Figura 02).

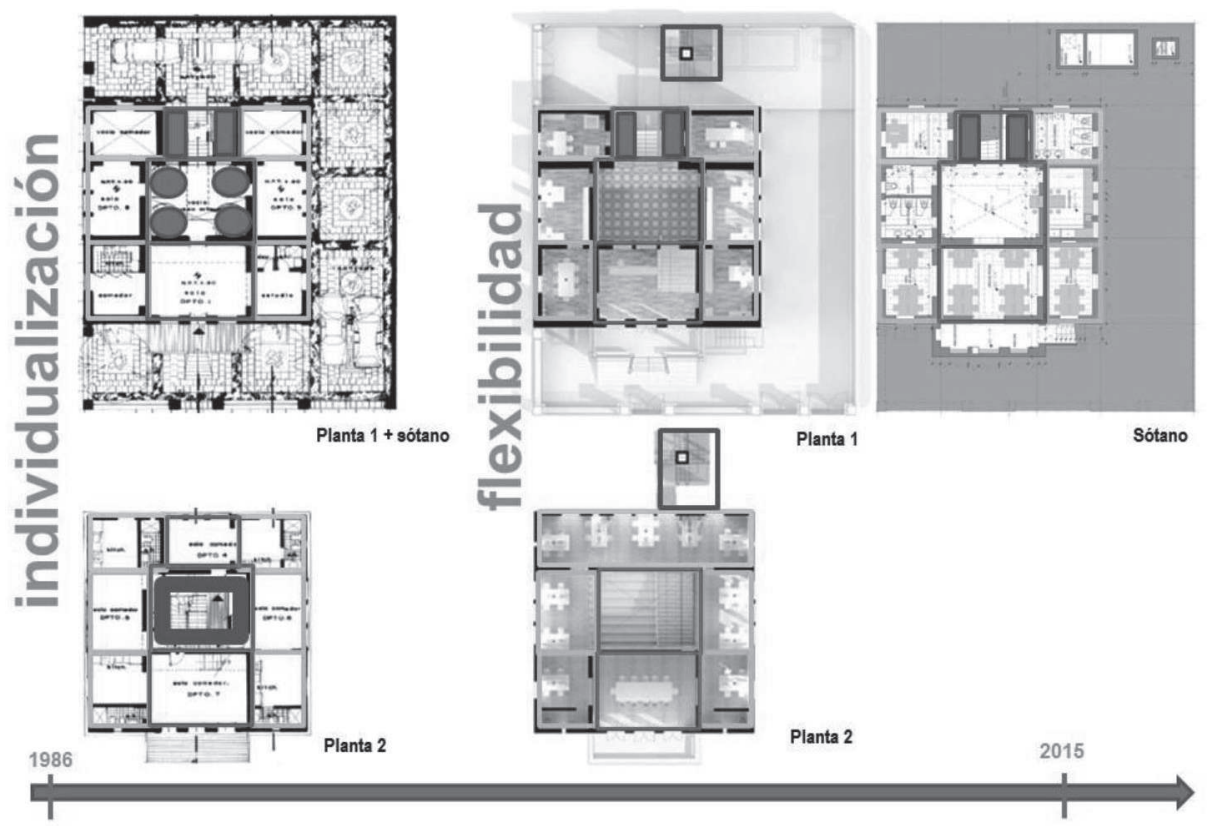

Figura 02. Características de las intervenciones de 1986 y 2015 que favorecieron la capacidad de reciclaje del edificio

El proyecto de oficinas tenía posibilidades futuras y se centró más en la capacidad de adaptación del espacio. El reciclaje arquitectónico es un proceso que incide directamente sobre el uso y no solo sobre la recuperación física del edificio. En la nueva propuesta se pudo ver esto y ejecutarlo.

Las primeras transformaciones que tuvo la Casa Apesteguía no están documentadas, pero se sabe que en un primer momento el edificio fue un rancho de una sola planta. En un segundo momento, indeterminado, el edificio se expandió hacia una segunda planta, como área de recreo y mirador. El autor estima que esta acción fue el punto de inicio para que la casa empezara a transformarse, de manera permanente y constante, a través del tiempo.

Ya en 1904, con la consolidación de la segunda planta, se evidenció la modulación de la estructura de adobe en 
la primera planta y el levantamiento de muros de quincha en la segunda planta. El espacio contenido del edificio adoptó un carácter de vivienda bifamiliar, con esquema tripartito $\mathrm{y}$ ambientes vinculados entre sí.

Con la remodelación de 1986 sucedió algo importante, pues la casa pasó de un carácter bifamiliar a uno multifamiliar, con la inclusión de un museo (Figura 03). La modulación de la estructura de la casa se siguió respetando, y esto se reflejó al momento de incorporar un sótano para albergar el museo, lo que conllevó a colocar muros de calza de concreto armado. Esto modificó la estructura de la casa, tal como se conocía hasta el momento, y quizás se le otorgó mayor estabilidad y durabilidad estructural en el tiempo. Además, se colocaron circulaciones interiores: mezanines y escaleras de madera y de fierro, que se descolgaban como volúmenes autónomos en la crujia central de triple altura para el ingreso a los departamentos. En la segunda planta se colocaron nuevas divisiones, en tanto que otras divisiones se desinstalaron, aunque se mantuvo la modulación que había poseído hasta ese momento.

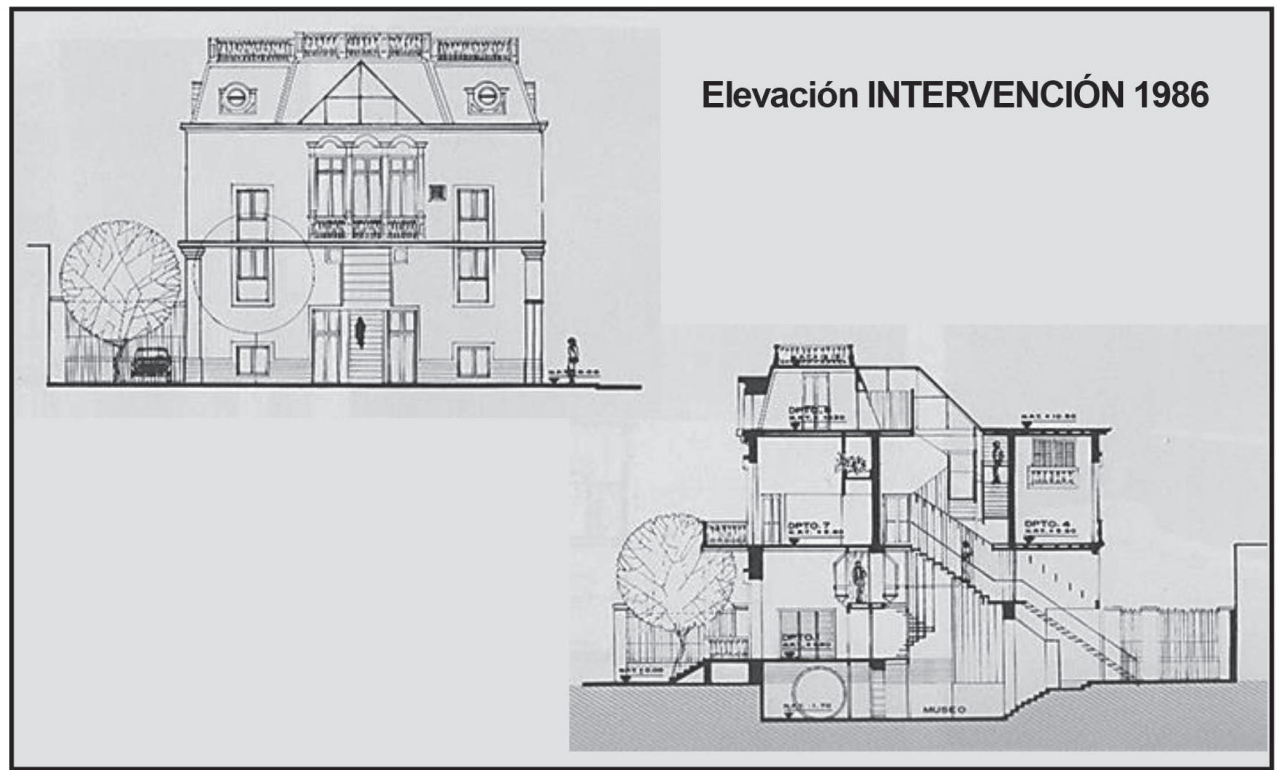

Corte INTERVENCIÓN 1986

Figura 03. Intervención en la Casa Apesteguía en 1986. La propuesta sirvió de base para el proyecto actual de oficinas para la agencia Fahrenheit DDB 
Posteriormente, hubo un breve lapso del cual no se sabe mucho, donde la casa cambió de uso y se convirtió en un hotel, pero mantuvo la estructura del espacio contenedor de la casa. Este hecho revela que el reciclaje arquitectónico está más ligado al uso, y no tan solo a las transformaciones físicas del edificio. Sin embargo, el edificio entró en obsolescencia (Rondinel, 2008, p. 56) y no necesariamente por su mal funcionamiento físico como edificio, sino por su deficiencia en comparación con lo que se ofrecía en el mercado. Es decir, cayó en desuso frente a las condiciones de vida existentes en esa época en inmuebles similares del entorno, razón por la cual se le abandonó. Hubo una mala adaptación de un uso residencial a un uso comercial.

Sin embargo, gracias a esa situación la casa Apesteguia pudo ser reciclada en el año 2015, pues no estaba dañada ni tampoco se presentaba la situación que no funcionara (FAUAUNI, 1993). Podía usarse de otra manera o reciclarse, para que funcionara adecuadamente. En ese año la agencia de publicidad Fahrenheit DDB compró el inmueble, que ya estaba declarado como patrimonio monumental. E1 edificio se encontraba en un estado de conservación aceptable, manteniendo su estructura con muros de adobe y quincha, revestidos de cal y yeso; con techos y cubiertas de madera y las instalaciones sanitarias y eléctricas en estado operativo.

El caso es que el uso de oficinas dispuesto, obligó a pensar de una manera totalmente diferente. "El mayor reto fue entender que la subdivisión de la casa en 8 departamentos hacía imposible un funcionamiento fluido de las dinámicas de trabajo y que resultaba necesario reorganizar el espacio en una sola unidad" (Masunoestudio, 2017). Se desmontaron los mezanines y escaleras de madera y fierro de la remodelación de 1986, revelando los espacios de altura original. Se reutilizó el eje vertical creado por el arquitecto Baracco en la intervención anterior, como un vacío que organiza el espacio y plantear allí una escalera/gradería que comunicará la primera y segunda planta (Figura 04 y 05). 

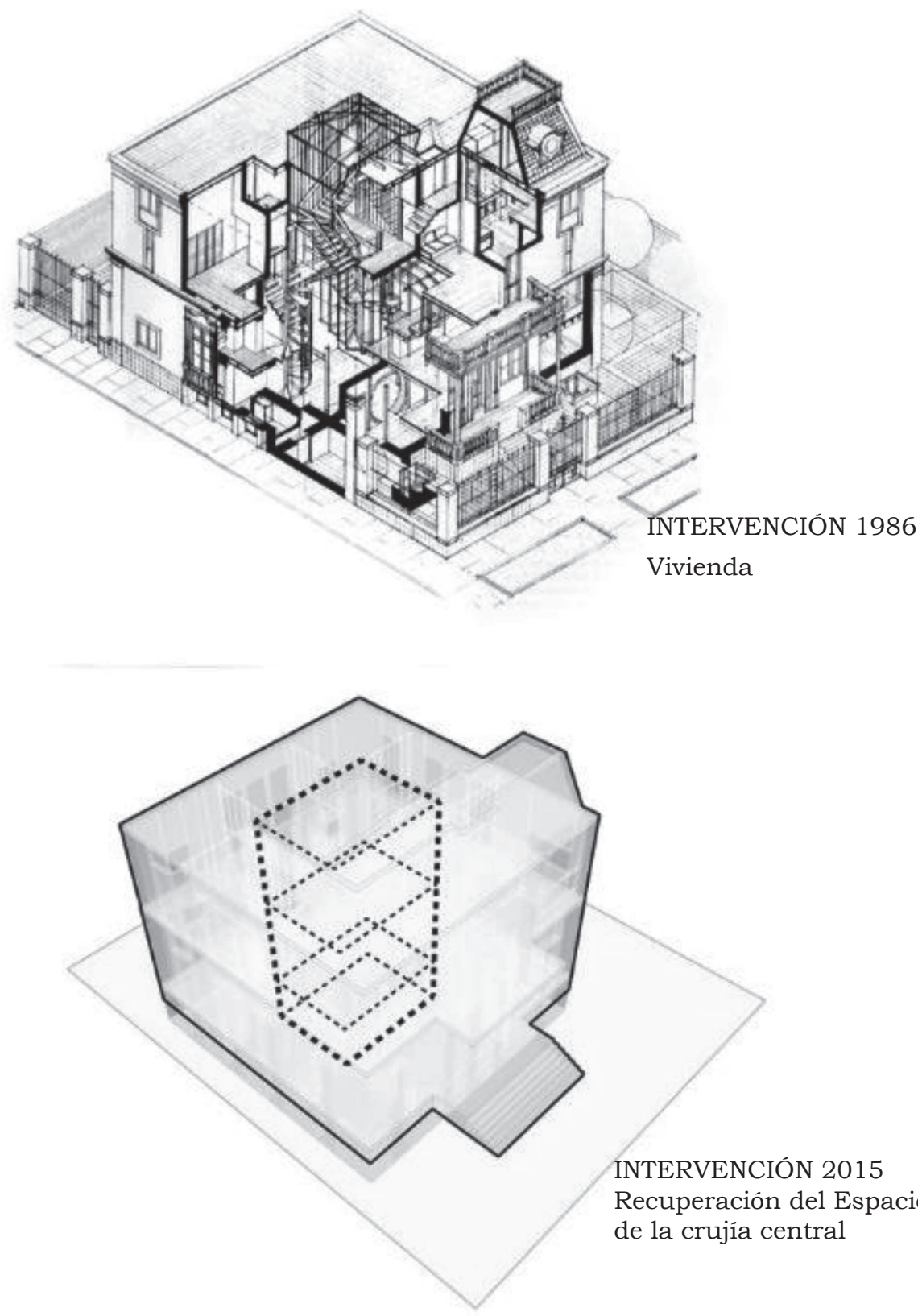

INTERVENCIÓN 2015

Recuperación del Espacio de la crujía central

Figura 04. Partida del concepto espacial de la intervención en la Casa Apesteguía en 2015 


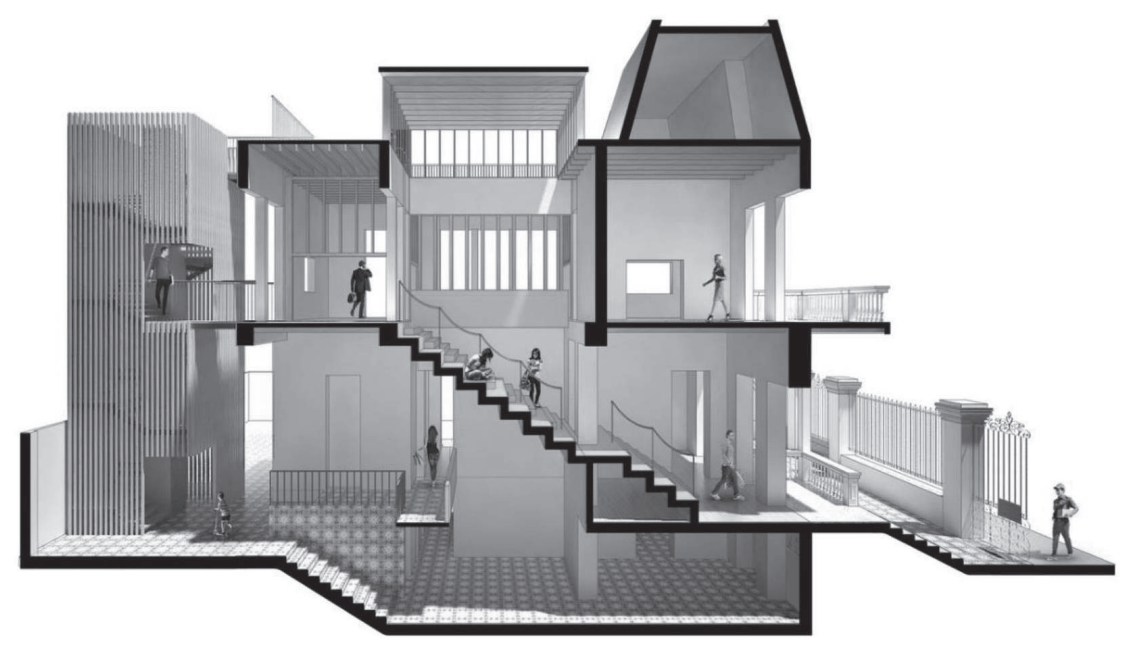

Figura 05. Corte fugado de la intervención en la Casa Apesteguia en 2015. La escalera/gradería hace espacio

Gracias a la flexibilidad, desmontabilidad, disgregabilidad y convertibilidad del material con que está formada la segunda planta: quincha, se pudo adecuar el uso al espacio. Para eso, se desmontaron algunos muros y se conservaron algunos pies derechos, logrando así una espacialidad más fluida. Esta situación permitió que la casa Apesteguía se adaptara a los nuevos propósitos

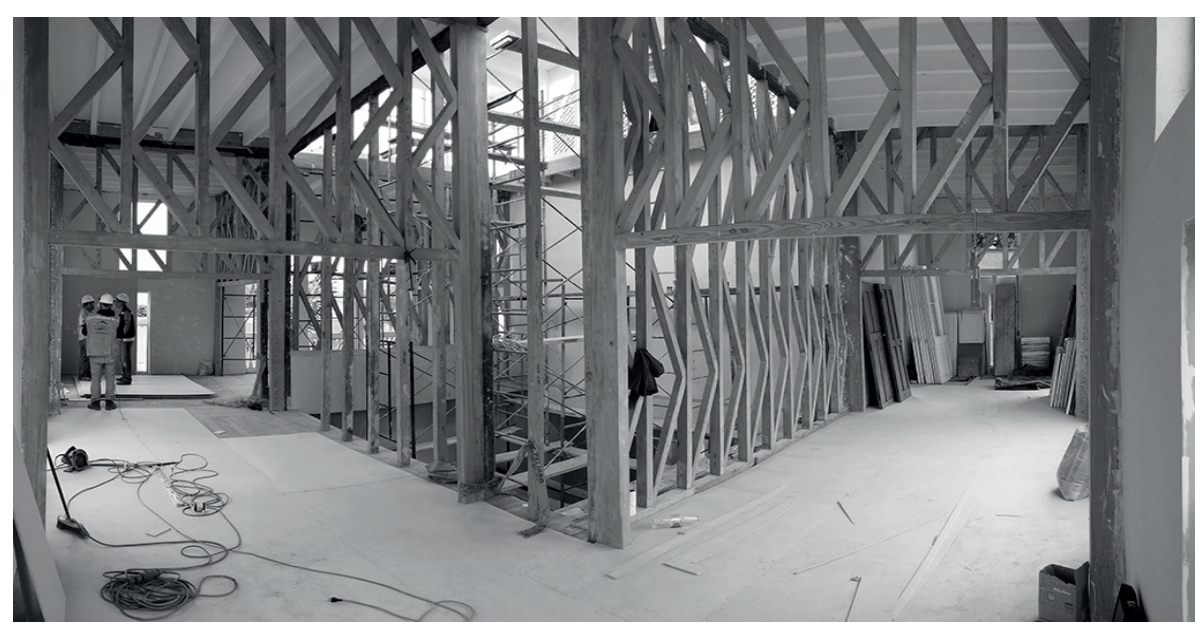

Figura 06. Desmontabilidad de los muros de la segunda planta. Vista de los pies derechos 
Quizás no se tuvo el concepto de capacidad de reciclaje en el momento de sus intervenciones, pero las incidencias de cambio que se suscitaron incidieron más en el cambio de uso, y de manera mínima las intervenciones en la estructura y tabiquería. De esta manera, se eliminó la obsolescencia del edificio y se logró que obtuviera muchas vidas más (Martucelli, 2008, p. 95).

\section{Conclusiones}

Tras la consulta de artículos y libro sobre el reciclaje de edificios, trabajos como de Callejo (2014) y Rondinel (2008), mencionan que la capacidad de reciclaje se entendería como la facultad de transformación de un edificio para albergar nuevos usos con el mínimo esfuerzo constructivo posible. Factores como la flexibilidad, la modulación y la individualización de los diferentes elementos constructivos (la estructura, la envolvente, los servicios y los tabiques), son fundamentales para que los edificios tengan potencial para el reciclaje. Por tanto, el empleo del reciclaje arquitectónico debería ser aplicado o descartado según la capacidad de reciclaje del edificio. Así, basta con tener una buena estructura del espacio contenedor y un espacio contenido aceptable para que el proyecto se presente como candidato al reciclaje.

Así lo demostró la casa Apesteguía, que durante más de un siglo ha permanecido en la ciudad, produciéndose transformaciones en la estructura del espacio contenedor, de acuerdo a los nuevos usos que se aplicaban en el espacio en su momento. Los muros perimetrales y algunas tabiquerias se mantuvieron; otras tabiquerias desaparecieron o se transformaron. Algunos vanos desaparecieron y otros aparecieron para dar lugar a una mayor fluidez espacial interior; pero siempre se respetó la modulación estructural. Se mantuvo la forma y el buen estado de su estructura, lo que permitió una flexibilidad espacial para albergar los nuevos usos, que fueron los que verdaderamente se reciclaron en el tiempo.

Los materiales de estos elementos también fueron un factor a favor de la desmontabilidad, porque al tratarse de madera y fierro no comprometían a la estructura del edificio. Habría que realizar un detallado estudio estructural, para considerar si la formación del sótano con muros de concreto que se hizo en 1986 influyó de manera favorable a la estabilidad y durabilidad de la estructura de adobe del edificio. Sin embargo, la permanencia de los materiales de construcción de la casa y algunos materiales compatibles con ellos de naturaleza similar, con predisposición de reciclaje, favoreció a su vigencia en el tiempo. La incorporación de materiales como el concreto armado a este tipo de edificaciones le hubiera quitado su adaptabilidad, su flexibilidad y su capacidad de reciclaje de residuos de la construcción.

Todos estos factores garantizaron que la casa Apesteguía se haya podido reciclar y comenzara nuevos ciclos de 
vida, cuyas dinámicas interiores impactaron de manera positiva a su entorno urbano inmediato. Es relevante este tipo de investigación en edificios antiguos de la ciudad para identificar posibles candidatos a proyectos de reciclaje y aminorar el impacto de construcciones en el entorno, ocupando edificios existentes de manera adecuada. Se debe reconocer que el proceso de reciclaje arquitectónico no es una estrategia actual, sino que es un hecho tan antiguo y propio como la arquitectura misma. Es por esta afirmación que la casa Apesteguia duró en el tiempo, sin tener en cuenta esas consideraciones teóricas en sus diferentes intervenciones. Aquel antiguo rancho que ha permanecido hasta la actualidad por un adecuado mantenimiento de la estructura y uso, podría aún durar mucho más en el tiempo.

\section{BIBLIOGRAFÍA}

Callejo, M. (2014). Reciclaje Arquitectónico: Definición, historia y capacidad. Universidad Politécnica de Valencia. Recuperado de https://riunet.upv.es/handle/10251/43647

Chacón, E. (2008). Software de reciclaje. Aproximación al diseño de programas para la transformación de la vivienda social. Congreso Internacional Sustainable Building 2010. Recuperado de http://www.sb10mad.com/ponencias/archivos/a/A048.pdf Facultad de Arquitectura, Urbanismo y Artes - Universidad Nacional de Ingeniería (1993). Arquitectura Republicana de Lima. Casa Apesteguia. Recuperado de http:// arquitecturalimarepublicana.blogspot.pe/2012/08/3104-casa-apesteguia.html Jourda, F. (2009). Petit manuel de la conception durable. Paris: Archibooks,.

Martínez, M. (2012). "Reciclaje de arquitectura vs restauración arquitectónica, cherramientas contrapuestas?”, en: Hábitat y Sociedad, 5, 23-33. Recuperado de http:// acdc.sav.us.es/habitatysociedad/images/stories/N05/N05A02\%20

Reciclaje\%20versus\%20restauracion.pdf

Martuccelli, E. (2008). "Reciclar y renovar. No una sino muchas vidas", en: Arquitextos $\mathrm{N}^{\circ} 23$. Lima.

Masunoestudio (2017). Oficinas Fahrenheit DDB. Recuperado de http://www.masunostudio.com/proyecto/oficinas-fahrenheit-ddb/

Rondinel, D. (2008). Arquitectura, reciclaje y sostenibilidad. Arquitextos $\mathrm{N}^{\circ} 23$. Lima.

Valdivia, R. (2008).RE + RE RE-novación y RE-ciclaje para el siglo XXI. Arquitextos №23. Lima. 